\title{
Whitney extension operators without loss of derivatives
}

\author{
Leonhard Frerick, Enrique Jordá and Jochen Wengenroth
}

\begin{abstract}
For a compact set $K \subseteq \mathbb{R}^{d}$ we characterize the existence of a linear extension operator $E: \mathscr{E}(K) \rightarrow \mathscr{C}^{\infty}\left(\mathbb{R}^{d}\right)$ for the space of Whitney jets $\mathscr{E}(K)$ without loss of derivatives, that is, it satisfies the best possible continuity estimates

$$
\sup \left\{\left|\partial^{\alpha} E(f)(x)\right|:|\alpha| \leq n, x \in \mathbb{R}^{d}\right\} \leq C_{n}\|f\|_{n},
$$

where $\|\cdot\|_{n}$ denotes the $n$-th Whitney norm. The characterization is by a surprisingly simple purely geometric condition introduced by Jonsson, Sjögren, and Wallis: there is $\varrho \in(0,1)$ such that, for every $x_{0} \in K$ and $\varepsilon \in(0,1)$, there are $d$ points $x_{1} \ldots, x_{d}$ in $K \cap B\left(x_{0}, \varepsilon\right)$ satisfying $\operatorname{dist}\left(x_{n+1}\right.$, affine hull $\left.\left\{x_{0}, \ldots, x_{n}\right\}\right) \geq \varrho \varepsilon$ for all $n \in\{0, \ldots, d-1\}$.
\end{abstract}

\section{Introduction}

The problem that compact sets $K \subseteq \mathbb{R}^{d}$ are often too small to determine all derivatives of differentiable functions on it was overcome by Whitney's [19] ingenious invention of spaces $\mathscr{E}^{n}(K)$ and $\mathscr{E}(K)=\mathscr{E}^{\infty}(K)$ of jets (of finite and infinite order, respectively), which he proved to be exactly the spaces of restrictions $\left(\left.\partial^{\alpha} f\right|_{K}\right)_{\alpha}$ for $f \in \mathscr{C}^{n}\left(\mathbb{R}^{d}\right), n \in \mathbb{N} \cup\{\infty\}$. In the finite order case, the extension can be even done by a continuous linear operator, and the last eighty years have seen countless results about the notoriously difficult problem to characterize the existence of continuous linear extension operators $\mathscr{E}(K) \rightarrow \mathscr{C}^{\infty}\left(\mathbb{R}^{d}\right)$ in the infinite order case if both spaces are endowed with their natural families of semi-norms, namely $|f|_{n}=\sup \left\{\left|\partial^{\alpha} f(x)\right|: x \in \mathbb{R}^{d},|\alpha| \leq m\right\}$ (which we will use only for functions with compact support so that the supremum is finite) on $\mathscr{C}^{\infty}\left(\mathbb{R}^{d}\right)$ and the Whitney norms introduced below for $\mathscr{E}(K)$.

In the present article we characterize the existence of operators which, simultaneously for all $n \in \mathbb{N}_{0} \cup\{\infty\}$, are extensions $\mathscr{E}^{n}(K) \rightarrow \mathscr{C}^{n}\left(\mathbb{R}^{d}\right)$. This is a situation

Mathematics Subject Classification (2010): 47A57.

Keywords: Whitney jets, extension operator. 
where a naive approach to solve certain problems (like partial differential equations) on $\mathscr{E}(K)$ works perfectly: extend the jet to $\mathbb{R}^{d}$, apply methods for smooth functions on $\mathbb{R}^{d}$, and restrict back to $K$.

Till now, only very few cases were understood, the most prominent result being Stein's [17] extension operator for sets with Lipschitz boundary. In view of the apparent difficulty of the unrestricted case and to our own surprise the final answer for the case of extension operators without loss of derivatives is the strikingly simple condition given in the abstract. Before stating this explicitly we provide the precise definitions and some background information.

\subsection{Whitney jets and extension operators}

For a compact set $K \subseteq \mathbb{R}^{d}$ we denote by $\mathscr{E}^{n}(K)$ and $\mathscr{E}(K)=\mathscr{E}^{\infty}(K)$ the spaces of Whitney jets of finite and infinite order, respectively, that is, families $f=$ $\left(f^{(\alpha)}\right)_{|\alpha|<n+1}$ of continuous (real or complex valued) functions whose formal Taylor polynomials (for finite $n$ )

$$
T_{y}^{n}(f)(x)=\sum_{|\alpha| \leq n} \frac{f^{(\alpha)}(y)}{\alpha !}(x-y)^{\alpha}
$$

give the "correct" approximation as if $f^{(\alpha)}$ were the partial derivatives of order $\alpha$, namely, the local "approximation errors"

$$
q_{n, t}(f)=\sup \left\{\frac{\left|f^{(\alpha)}(x)-\partial^{\alpha} T_{y}^{n}(f)(x)\right|}{|x-y|^{n-|\alpha|}}:|\alpha| \leq n, x, y \in K, 0<|x-y| \leq t\right\}
$$

tend to 0 for $t \rightarrow 0$ (and all $n$ in the case of $\mathscr{E}(K)$ ). The $n$-th Whitney norm is then

$$
\|f\|_{n}=\sup \left\{\left|f^{(\alpha)}(x)\right|: x \in K,|\alpha| \leq n\right\}+\sup \left\{q_{n, t}(f): t>0\right\} .
$$

Clearly, Taylor's theorem implies that for any $n \in \mathbb{N}_{0} \cup\{\infty\}$ and $g \in \mathscr{C}^{n}(\Omega)$ with an open set $\Omega \supseteq K$ the restrictions $R(g)=\left(\left.\partial^{\alpha} g\right|_{K}\right)_{|\alpha|<n+1}$ are jets of order $n$. A celebrated result of Whitney [19] says that each jet $f \in \mathscr{E}^{n}(K)$ has such an extension. He even proved that one can extend jets of finite order by a continuous linear operator. Whitney's methods yield that $\mathscr{E}(K)$ is always a Fréchet space isomorphic to a quotient of $\mathscr{C}^{\infty}\left(\mathbb{R}^{d}\right)$ where the restriction operator $R$ is the quotient map. Moreover, the $n$-th Whitney norm on $K$ is equivalent to the $n$-th quotient semi-norm $\inf \left\{|g|_{n}: R(g)=f\right\}$ (which follows from from Whitney's work or, e.g., the method to prove Theorem I.4.1 in Malgrange's book [9]).

The existence of a continuous linear extension operator for jets of infinite order depends on the shape of the compact set and there is a vast amount of literature about this question, we refer to the introduction of [6] for an overview. In particular, if $K$ is too small, e.g. a singleton (in which case Whitney's theorem was already shown by Borel [2]), Mitjagin [11] observed that there is no continuous linear extension operators (although there is always a linear but discontinuous one, as well as a continuous but non-linear one - just because this is always so for quotients of Fréchet spaces, see e.g. Bourbaki's book on topological vector spaces, [4], 
Chapter II, §4). On the other hand, there are several results proving that there is a continuous linear extension operator if $K$ is "big enough", we only mention the work of Tidten [18].

\subsection{Extensions without loss of derivatives}

The problem we deal with in this paper is the characterization of compact sets $K$ having an extension operator $E: \mathscr{E}(K) \rightarrow \mathscr{C}^{\infty}\left(\mathbb{R}^{d}\right)$ which induces, simultaneously for all $n$, continuous extension operators $E: \mathscr{E}^{n}(K) \rightarrow \mathscr{C}^{n}\left(\mathbb{R}^{d}\right)$. Since $\mathscr{E}(K)$ is dense in $\mathscr{E}^{n}(K)$ this is the equivalent to the continuity estimates

$$
\sup \left\{\left|\partial^{\alpha} E(f)(x)\right|:|\alpha| \leq n, x \in \mathbb{R}^{d}\right\} \leq C_{n}\|f\|_{n} \text { for all } n \in \mathbb{N}_{0} .
$$

We therefore say that $E$ is an extension operator without loss of derivatives.

Seeley [16] gave a simple construction of such operators for half spaces, and Stein [17] found extension operators without loss of derivatives for compact subsets $K$ with Lip $_{1}$-boundary. Also Rogers [15] gave a sufficient geometric condition for $K$ permitting the existence of an operator such that even all the Sobolev spaces $W_{k}^{p}(K)$ can be extended to $W_{k}^{p}\left(\mathbb{R}^{d}\right)$ for each $k \in \mathbb{N}$ and $1 \leq p \leq \infty$.

Our main result will use a (local) Markov inequality for polynomials in a form (apparently) first used by Jonsson, Sjögren, and Wallin [8]. Pawłucki and Pleśniak [12], [13], as well as Pleśniak [14], used a global version to characterize the existence of continuous linear extension operators for $\mathscr{E}(K)$ with a weaker topology (which, of course, implies continuity with respect to the Whitney norms), and Bos and Milman [3] introduced a local Markov inequality with some exponent $r \geq 1(\mathrm{LMI}(r))$ on a compact set $K$ (which eventually turned out to be equivalent to Pawłucki's and Pleśniak's condition) to obtain extension operators with homogeneous loss of differentiability. The exact loss of differentiability was then characterized in [7].

Let us now give the precise definition. $K \subseteq \mathbb{R}^{d}$ satisfies the $\operatorname{LMI}(r)$ if there exist $\varepsilon_{0}>0$ and constants $c_{k} \geq 1$ such that for each polynomial $P$ of degree $\operatorname{deg}(P) \leq k$, each $\varepsilon \in\left(0, \varepsilon_{0}\right)$, and each $x_{0} \in K$ we have

$$
\left|\nabla P\left(x_{0}\right)\right| \leq c_{k} \varepsilon^{-r}\|P\|_{B\left(x_{0}, \varepsilon\right) \cap K}
$$

where $\|\cdot\|_{M}$ denotes the uniform norm on a set $M \subseteq \mathbb{R}^{d}$ and $B\left(x_{0}, \varepsilon\right)$ the closed ball of radius $\varepsilon$ centered at $x_{0}$.

By applying the estimate $k$-times with $2^{-k} \varepsilon$ instead of $\varepsilon$ we obtain (with different constants $c_{k}$ ) for all $\alpha \in \mathbb{N}_{0}^{d}$,

$$
\left|\partial^{\alpha} P\left(x_{0}\right)\right| \leq c_{k} \varepsilon^{-r|\alpha|}\|P\|_{B\left(x_{0}, \varepsilon\right) \cap K},
$$

which is $\operatorname{LMI}(r)$ in the form used by Bos and Milman.

Modifying the constants in the definition of LMI one can replace the existence quantifier for $\varepsilon_{0}$ by the universal quantifier or just by $\varepsilon_{0}=1$. (Bos and Milman kept track of the constants and therefore, the formulation with $\varepsilon_{0}$ was used, for our purpose, the constants are not important). 
LMI measures the "local size" of $K$ near its boundary points and will serve as the characterization of the existence of extension operators without loss of derivatives in our main contribution:

Theorem 1. A compact set $K \subseteq \mathbb{R}^{d}$ has an extension operator $\mathscr{E}(K) \rightarrow \mathscr{C}^{\infty}\left(\mathbb{R}^{d}\right)$ without loss of derivatives if and only if $K$ satisfies LMI(1).

The big advantage of LMI(1) compared to the case $r>1$ is that Jonsson, Sjögren, and Wallin (Theorems 1.2 and 1.3 in [8]) showed that it is enough to check LMI(1) for polynomials of degree 1 which enabled them to obtain a purely geometric characterization figuring in the following corollary.

Corollary 2. A compact set $K \subseteq \mathbb{R}^{d}$ has an extension operator $\mathscr{E}(K) \rightarrow \mathscr{C}^{\infty}\left(\mathbb{R}^{d}\right)$ without loss of derivatives if and only if there is $\varrho \in(0,1)$ such that, for every $x_{0} \in K$ and $\varepsilon \in(0,1), K \cap B\left(x_{0}, \varepsilon\right)$ is not contained in any band of the form $\left\{x \in \mathbb{R}^{d}:\left|\left\langle b, x-x_{0}\right\rangle\right| \leq \varrho \varepsilon\right\}$, where $b \in \mathbb{R}^{d}$ is any vector of norm 1 .

An alternative formulation of the condition was given by Bos and Milman in Theorem D of [3]: $K \subseteq \mathbb{R}^{d}$ satisfies $\operatorname{LMI}(1)$ if and only if there is $\varrho \in(0,1)$ such that for every $x_{0} \in K$ and $\varepsilon \in(0,1)$ there are $d$ points $x_{1} \ldots, x_{d}$ in $K \cap B\left(x_{0}, \varepsilon\right)$ such that for all $n \in\{0, \ldots, d-1\}$

$$
\operatorname{dist}\left(x_{n+1}, \text { affine hull }\left\{x_{0}, \ldots, x_{n}\right\}\right) \geq \varrho \varepsilon
$$

(the equivalence of this condition to the one of Jonsson, Sjögren, and Wallin is elementary).

These geometric conditions are very easy to check in concrete cases so that, for instance, our result includes Stein's theorem about compact sets with Lip $1^{-}$ boundary as well as sets with inward directed cusps (which are covered neither by Stein's theorem nor by Roger's results). Moreover, we easily obtain that such porous sets like Cantor's or the Sierpiński triangle admit extension operators without loss of differentiability whereas sets with outward directed cusps do not.

\subsection{The trace space}

Let us close this introduction with the remark that there is of course an alternative approach to smooth functions on small sets just by setting $\mathscr{C}^{\infty}(K)=\left\{\left.f\right|_{K}: f \in\right.$ $\left.\mathscr{C}^{\infty}\left(\mathbb{R}^{d}\right)\right\}$ endowed with the quotient topology. If $\mathscr{E}(K)$ admits a linear continuous extension operator at all then, by a result in [6], remark $3.13, \mathscr{E}(K)$ and $\mathscr{C}^{\infty}(K)$ coincide (more precisely, $\left(f^{(\alpha)}\right)_{\alpha \in \mathbb{N}_{0}^{d}} \mapsto f^{(0)}$ is an isomorphism) and our result thus applies to the latter space. Instead of a continuous linear extension the ideas in [6] yield that it would be enough that for all $n \in \mathbb{N}$ there are $m(n) \in \mathbb{N}$ and $C(n)>0$ such that for every $f \in \mathscr{E}(K)$ there is an extension $F \in \mathscr{C}^{\infty}\left(\mathbb{R}^{d}\right)$ with $|F|_{n} \leq C(n)\|f\|_{m(n)}$. In particular, a continuous and homogeneous extension map would be enough.

However, if $K$ is too small, $\mathscr{C}^{\infty}(K)$ and $\mathscr{E}(K)$ are very different spaces (consider, for example, the extreme case of a singleton) and much less is known about 
extension operators for $\mathscr{C}^{\infty}(K)$. On the one hand, there is a very deep result of Bierstone and Milman [1] about semicoherent subanalytic sets; and on the other hand, two special cases of non-subanalytic sets were treated by Fefferman and Ricci in [5].

\subsection{Necessity of LMI}

The main point of our contribution is certainly the sufficiency of LMI(1) and, thus, the geometric conditions of Jonsson, Sjögren, and Wallin, and Bos and Milman, respectively. Necessity of LMI(1) follows from the methods in [3] and a short explicit proof is also contained in [7]. We thus only describe very briefly the main idea in [3]: The key ingredients are the classical Sobolev-Gagliardo-Nirenberg (SGN) inequalities with optimal exponents (which, in terms of the structure theory of Fréchet spaces, correspond to Vogt's condition (DN), see, e.g., Meise and Vogt [10]) which hold for smooth functions on a large ball containing $K$ and just by applying them to $E(f)$ for $f \in \mathscr{E}(K)$ one gets the SGN inequalities for $\mathscr{E}(K)$. To obtain then LMI for polynomials one multiplies with a bump function, applies SGN inequalities and uses an iterative application of the estimates so obtained.

The rest of the paper is organized as follows. In section 2 we will explain the construction of the extension operator which is based on certain measures $\mu_{\alpha}$ so that $\int f d \mu_{\alpha}$ interpolate the partial derivatives (this part is similar to our previous article [7] and was inspired by Whitney's original construction). In the third section we will then show how to obtain those measures if $K$ satisfies LMI(1).

\section{Construction of the extension operators}

We first recall Whitney's explicit construction of an extension operator for $\mathscr{E}^{n}(K)$. For a suitable partition of unity $\left(\varphi_{i}\right)_{i \in \mathbb{N}}$ of $\Omega \backslash K$ (where $\Omega$ is an open set containing $K$ ) such that the supports of $\varphi_{i}$ tend to $K$, and for $x_{i} \in K$ minimizing the distance to $\operatorname{supp}\left(\varphi_{i}\right)$ the operator $E_{n}$ is of the following form:

$$
E_{n}(f)(x)= \begin{cases}f^{(0)}(x), & x \in K, \\ \sum_{i=1}^{\infty} \varphi_{i}(x) T_{x_{i}}^{n}(f)(x), & x \notin K .\end{cases}
$$

As we want to have an operator which works simultaneously for all $n \in \mathbb{N}_{0}$ we replace the $n$-th degree Taylor polynomials around $x_{i}$ by certain "interpolations" which only depend on $f^{(0)}$, namely

$$
S_{i}(f)(x)=\sum_{|\alpha| \leq i} \frac{1}{\alpha !} \mu_{\alpha, i}\left(f^{(0)}\right)\left(x-x_{i}\right)^{\alpha},
$$

where $\mu_{\alpha, i}\left(f^{(0)}\right)=\int f^{(0)} d \mu_{\alpha, i}$ is the integral of $f$ with respect to a suitable (complex) measure. 
Theorem 3. Suppose that for all $\alpha \in \mathbb{N}_{0}^{d}, x \in \partial K$, and $\varepsilon>0$ there are measures $\nu_{\alpha, x, \varepsilon}$ on $K$ such that, for all $n \in \mathbb{N}_{0}$ and $f \in \mathscr{E}^{n}(K)$,

$$
\begin{aligned}
& \lim _{\varepsilon \rightarrow 0} \sup _{|\alpha| \leq n, x \in \partial K} \frac{\left|\nu_{\alpha, x, \varepsilon}\left(f^{(0)}\right)-\varepsilon^{|\alpha|} f^{(\alpha)}(x)\right|}{\varepsilon^{n}}=0 \quad \text { and } \\
& \lim _{\varepsilon \rightarrow 0} \sup _{|\alpha|>n, x \in \partial K} \frac{\left|\nu_{\alpha, x, \varepsilon}\left(f^{(0)}\right)\right|}{\varepsilon^{n}}=0 .
\end{aligned}
$$

Then $K$ has an extension operator without loss of derivatives.

Proof. We consider the partition of unity constructed by Whitney [19]. For $K \subseteq \mathbb{R}^{d}$ compact there are an open set $\Omega$ containing $K$ and positive test functions $\varphi_{i} \in$ $\mathscr{D}(\Omega \backslash K)$ with the following properties:

(i) $\sum_{i=1}^{\infty} \varphi_{i}(x)=1$ for all $x \in \Omega \backslash K$ and each point belongs to at most $N$ $\operatorname{supports} \operatorname{supp}\left(\varphi_{i}\right)$ for some constant $N \in \mathbb{N}$.

(ii) $\operatorname{supp}\left(\varphi_{i}\right) \rightarrow K$ for $i \rightarrow \infty$, that is, for each $\varepsilon>0$ there is $k \in \mathbb{N}$ such that $\operatorname{supp}\left(\varphi_{i}\right) \subseteq\left\{x \in \mathbb{R}^{d}: \operatorname{dist}(x, K)<\varepsilon\right\}$ for all $i \geq k$.

(iii) $\operatorname{diam}\left(\operatorname{supp}\left(\varphi_{i}\right)\right) \leq 2 \operatorname{dist}\left(\operatorname{supp}\left(\varphi_{i}\right), K\right)$ (where diam is the diameter of a set).

(iv) There are constants $c_{\beta}$ such that $\left|\partial^{\beta} \varphi_{i}(x)\right| \leq c_{\beta} \operatorname{dist}(x, K)^{-|\beta|}$ for all $i \in \mathbb{N}$, $\beta \in \mathbb{N}_{0}^{d}$, and $x \in \mathbb{R}^{d}$.

With the help of this partition, Whitney showed that the operators $E_{n}$ defined above are indeed continuous linear extension operators from $\mathscr{E}^{n}(K)$ to $\mathscr{C}^{n}\left(\mathbb{R}^{d}\right)$. Let us denote

$$
\gamma_{i}=\operatorname{dist}\left(K, \operatorname{supp}\left(\varphi_{i}\right)\right)=\operatorname{dist}\left(x_{i}, \operatorname{supp}\left(\varphi_{i}\right)\right) .
$$

For $|\beta| \leq n$ and $|\alpha| \leq n$ we can use Leibniz' rule, (iii), and (iv) to obtain positive constants $C_{n}$ independent of $i$ such that

$$
\left|\partial^{\beta}\left(\left(x-x_{i}\right)^{\alpha} \varphi_{i}(x)\right)\right| \leq C_{n} \gamma_{i}^{|\alpha|-|\beta|} \text { for all } x \in \operatorname{supp}\left(\varphi_{i}\right) .
$$

For $|\beta| \leq n$ and $|\alpha|>n$ we observe that property (iii) implies that $\left|x-x_{i}\right| \leq$ $3 \gamma_{i}$ for all $x \in \operatorname{supp}\left(\varphi_{i}\right)$. Using Leibniz's rule again, we find (different) $C_{n}$ not depending on $i$, such that

$$
\left|\partial^{\beta}\left(\left(x-x_{i}\right)^{\alpha} \varphi_{i}(x)\right)\right| \leq C_{n} 3^{|\alpha|} \sup _{\gamma \leq \beta, \gamma \leq \alpha} \frac{\alpha !}{(\alpha-\gamma) !} \gamma_{i}^{|\alpha|-|\beta|}, \quad x \in \operatorname{supp}\left(\varphi_{i}\right) .
$$

We remark that, for $|\beta| \leq n$,

$$
\begin{aligned}
\sum_{|\alpha|>n} \sup _{\gamma \leq \beta, \gamma \leq \alpha} \frac{1}{(\alpha-\gamma) !} 3^{|\alpha|} & \leq 3^{|\beta|} \sum_{\gamma \leq \beta} \sum_{|\alpha|>n, \alpha \geq \gamma} \frac{1}{(\alpha-\gamma) !} 3^{|\alpha-\gamma|} \\
& \leq 3^{n}(n+1)^{d}\left(\sum_{j \in \mathbb{N}_{0}} \frac{1}{j !} 3^{j}\right)^{d}=e^{3 d}(n+1)^{d} 3^{n}
\end{aligned}
$$


We set $\mu_{\alpha, i}=\nu_{\alpha, x_{i}, \gamma_{i}} / \gamma_{i}^{|\alpha|}$, and for $f \in \mathscr{E}^{0}(K)=\mathscr{C}(K)$ we define

$$
E(f)= \begin{cases}f^{(0)}(x), & x \in K \\ \sum_{i \in \mathbb{N}} \varphi_{i}(x) \sum_{|\alpha| \leq i} \frac{1}{\alpha !} \mu_{\alpha, i}\left(f^{(0)}\right)\left(x-x_{i}\right)^{\alpha}, & x \notin K\end{cases}
$$

With Whitney's operators $E_{n}$ we will show below that for all $|\beta| \leq n, f \in \mathscr{E}^{n}(K)$ we have

$$
\left|\partial^{\beta} E(f)(x)-\partial^{\beta} E_{n}(f)(x)\right|=o\left(\operatorname{dist}(x, K)^{n-|\beta|}\right) \quad \text { for } x \rightarrow \partial K \text {. }
$$

This implies that $\left(E-E_{n}\right)(f)$ admits derivatives up to order $n$ in (the boundary of) $K$ and that they all vanish on $K$. Since the partition $\varphi_{i}$ is locally finite $E(f)$ is clearly $\mathscr{C}^{\infty}$ on $\mathbb{R}^{d} \backslash K$. Thus $E-E_{n}: \mathscr{E}^{n}(K) \rightarrow \mathscr{C}^{n}\left(\mathbb{R}^{d}\right)$ is a well defined linear operator and takes its values in $\mathscr{J}^{n}(K)=\left\{g \in \mathscr{C}^{n}\left(\mathbb{R}^{d}\right)\right.$ : $\partial^{\alpha} g(x)=0$ for all $\left.x \in K,|\alpha| \leq n\right\}$.

It is clear that the operator is continuous if we consider in $\mathscr{J}^{n}(K)$ the topology of pointwise convergence in $\mathbb{R}^{d} \backslash K$. Since this topology is Hausdorff we can apply the closed graph theorem to conclude that $E-E_{n}: \mathscr{E}^{n}(K) \rightarrow \mathscr{J}^{n}(K)$ is continuous with respect to the Fréchet space topology on $\mathscr{J}^{n}(K)$, and therefore also $E: \mathscr{E}^{n}(K) \rightarrow \mathscr{C}^{n}\left(\mathbb{R}^{d}\right)$ is continuous.

Let us now prove (2.4). For $x \in \mathbb{R}^{d} \backslash K$ we define $i(x)=\min \{i \in I: x \in$ $\left.\operatorname{supp} \varphi_{i}\right\}$. Because of the property (ii) we then have $i(x) \rightarrow \infty$ if $x \rightarrow \partial K$. For $|\beta| \leq n, f \in \mathscr{E}^{n}(K)$, and $i(x)>n$ we have

$$
\begin{aligned}
\partial^{\beta}\left(E(f)-E_{n}(f)\right)(x)= & \sum_{i \geq i(x)} \sum_{|\alpha| \leq n} \frac{1}{\alpha !}\left(\mu_{\alpha, i}\left(f^{(0)}\right)-f^{(\alpha)}\left(x_{i}\right)\right) \partial^{\beta}\left(\left(x-x_{i}\right)^{\alpha} \varphi_{i}(x)\right) \\
& +\sum_{i \geq i(x)} \sum_{n<|\alpha| \leq i} \frac{1}{\alpha !} \mu_{\alpha, i}\left(f^{(0)}\right) \partial^{\beta}\left(\left(x-x_{i}\right)^{\alpha} \varphi_{i}(x)\right) .
\end{aligned}
$$

We will estimate both terms. Using the hypotheses on the measures we get, for $|\alpha| \leq n$,

$$
\left|\mu_{\alpha, i}\left(f^{(0)}\right)-f^{(\alpha)}\left(x_{i}\right)\right|=o\left(\gamma_{i}^{n-|\alpha|}\right) \quad \text { as } i \rightarrow \infty .
$$

From this, (2.1), and the bound for the number of supports that can contain $x$ we obtain

$$
\left|\sum_{|\alpha| \leq n} \frac{1}{\alpha !}\left(\mu_{\alpha, i}\left(f^{(0)}\right)-f^{(\alpha)}\left(x_{i}\right)\right) \partial^{\beta}\left(\left(x-x_{i}\right)^{\alpha} \varphi_{i}(x)\right)\right|=o\left(\gamma_{i}^{n-|\beta|}\right) \quad \text { for } i \rightarrow \infty,
$$

where the limit is uniform with respect to $x \in \mathbb{R}^{d}$.

From $\gamma_{i} \leq d(x, K) \leq 3 \gamma_{i}$ for each $x \in \operatorname{supp}\left(\varphi_{i}\right)$ it then follows that

$$
\lim _{x \rightarrow \partial K} \sum_{i \geq i(x)} \frac{1}{d(x, K)^{n-|\beta|}}\left|\sum_{|\alpha| \leq n} \frac{1}{\alpha !}\left(\mu_{\alpha, i}\left(f^{(0)}\right)-f^{(\alpha)}\left(x_{i}\right)\right) \partial^{\beta}\left(\left(x-x_{i}\right)^{\alpha} \varphi_{i}(x)\right)\right|=0 .
$$


For the second summand we use again the hypotheses to get

$$
\sup _{|\alpha|>n}\left|\mu_{\alpha, i}\left(f^{(0)}\right) \gamma_{i}^{|\alpha|}\right|=o\left(\gamma_{i}^{n}\right) \quad \text { as } i \rightarrow \infty .
$$

Then (2.2) and (2.3) imply that (again uniformly in $x$ )

$$
\begin{aligned}
\mid \sum_{|\alpha|>n} \frac{1}{\alpha !} \mu_{\alpha, i}\left(f^{(0)}\right) & \partial^{\beta}\left(\left(x-x_{i}\right)^{\alpha} \varphi_{i}(x)\right) \mid \\
& \leq \sum_{|\alpha|>n} \frac{1}{\alpha !} \gamma_{i}^{-|\alpha|} o\left(\gamma_{i}^{n}\right) C_{n} \sup _{\gamma \leq \beta, \gamma \leq \alpha} \frac{\alpha !}{(\alpha-\gamma) !} 3^{|\alpha|} \gamma_{i}^{|\alpha|-|\beta|} \\
& \leq o\left(\gamma_{i}^{n}\right) C_{n} e^{3 d}(n+1)^{d} 3^{n} \gamma_{i}^{-|\beta|}=o\left(\gamma_{i}^{n-|\beta|}\right) \quad \text { as } i \rightarrow \infty .
\end{aligned}
$$

Altogether we obtain (remembering $i(x) \rightarrow \infty$ if $x \rightarrow \partial K$ )

$$
\lim _{x \rightarrow \partial K} \sum_{i \geq i(x)} \frac{1}{d(x, K)^{n-|\beta|}}\left|\sum_{n<|\alpha| \leq i} \frac{1}{\alpha !} \mu_{\alpha, i}\left(f^{(0)}\right) \partial^{\beta}\left(\left(x-x_{i}\right)^{\alpha} \varphi_{i}(x)\right)\right|=0,
$$

which gives (2.4).

\section{Construction of the measures}

To finish the proof of the main theorem we have to show the existence of the measures figuring in Theorem 3:

Proposition 4. Let $K \subseteq \mathbb{R}^{d}$ be a compact subset satisfying $\mathrm{LMI}(1)$. For all $\alpha \in \mathbb{N}_{0}^{d}, x \in \partial K$, and $\varepsilon \in(0,1)$ there is a measure $\nu_{\alpha, x, \varepsilon}$ on $K$ such that, for all $n \in \mathbb{N}_{0}$ and $f \in \mathscr{E}^{n}(K)$,

$$
\begin{aligned}
& \lim _{\varepsilon \rightarrow 0} \sup _{|\alpha| \leq n, x \in \partial K} \frac{\left|\nu_{\alpha, x, \varepsilon}\left(f^{(0)}\right)-\varepsilon^{|\alpha|} f^{(\alpha)}(x)\right|}{\varepsilon^{n}}=0 \quad \text { and } \\
& \lim _{\varepsilon \rightarrow 0} \sup _{|\alpha|>n, x \in \partial K} \frac{\left|\nu_{\alpha, x, \varepsilon}\left(f^{(0)}\right)\right|}{\varepsilon^{n}}=0 .
\end{aligned}
$$

The main ingredient in the proof will be the solution of a suitable moment problem and in order to get uniform estimates for $x \in \partial K$ we will apply scaling arguments. It is therefore convenient to consider the following "blow-ups" of $K$ with respect to a boundary point:

$$
A_{x, \varepsilon}=\varepsilon^{-1}(K-x) \cup\left\{y \in \mathbb{R}^{d}:|y| \geq \varepsilon^{-1}\right\} .
$$

The union with the complement of the large ball is necessary to solve the moment problem. However, cutting off the part of the measures supported in $\left\{|y| \geq \varepsilon^{-1}\right\}$ will have no influence on the properties required in Theorem 3.

The moment problem is described in the following proposition. 
Proposition 5. Let $K \subseteq \mathbb{R}^{d}$ be a compact subset satisfying $\mathrm{LMI}(1)$. Then there exists a continuous and radial function $\varrho: \mathbb{R}^{d} \rightarrow(0, \infty)$ with $|y|^{n}=o(\varrho(y))$ for $|y| \rightarrow \infty$ and all $n \in \mathbb{N}$ such that for each $x \in \partial K, \varepsilon \in(0,1)$, and $\alpha \in \mathbb{N}_{0}^{d}$ there exists a finite regular Borel measure $\mu:=\mu_{\alpha, x, \varepsilon}$ on $A_{x, \varepsilon}$ with total variation $|\mu|\left(A_{x, \varepsilon}\right) \leq 1$ such that

$$
\int_{A_{x, \varepsilon}} y^{\beta} \frac{1}{\varrho(y)} d \mu(y)= \begin{cases}\alpha !, & \beta=\alpha \\ 0, & \text { else. }\end{cases}
$$

Before proving this let us show how to obtain Proposition 4.

Proof of Proposition 4. We assume without loss of generality that $K \subseteq B(0,1 / 4)$. For $x \in \partial K, \varepsilon \in(0,1), \alpha \in \mathbb{N}_{0}^{d}$, and $f \in \mathscr{C}(K)$ we define

$$
\nu_{\alpha, x, \varepsilon}(f)=\int_{\varepsilon^{-1}(K-x)} f(\varepsilon y+x) \frac{1}{\varrho(y)} d \mu_{\alpha, x, \varepsilon}(y),
$$

with $\mu_{\alpha, x, \varepsilon}$ from Proposition 5. For each $f \in \mathscr{C}\left(\mathbb{R}^{d}\right)$ with support in $B(0,3 / 4)$ we then have

$$
\nu_{\alpha, x, \varepsilon}\left(\left.f\right|_{K}\right)=\int_{A_{x, \varepsilon}} f(\varepsilon y+x) \frac{1}{\varrho(y)} d \mu_{\alpha, x, \varepsilon}(y)
$$

since $|x+\varepsilon y|>3 / 4$ whenever $|y|>1 / \varepsilon$.

Multiplying with a cut-off function we may assume that Whitney's extension operator $E_{n}: \mathscr{E}^{n}(K) \rightarrow \mathscr{C}^{n}\left(\mathbb{R}^{d}\right)$ has values in the space of $\mathscr{C}^{n}$-functions with support in $B(0,3 / 4)$. For $f \in \mathscr{E}^{n}(K)$ we denote by $F=E_{n}(f)$ an extension of $f$, and obtain from Taylor's theorem and the condition on the moments of $\mu_{\alpha, x, \varepsilon}$,

$$
\begin{aligned}
& \left|\nu_{\alpha, x, \varepsilon}\left(f^{(0)}\right)-\varepsilon^{|\alpha|} f^{(\alpha)}(x)\right|=\left|\int_{A_{x, \varepsilon}} F(\varepsilon y+x) \frac{1}{\varrho(y)} d \mu_{\alpha, x, \varepsilon}(y)-\varepsilon^{|\alpha|} \partial^{\alpha} F(x)\right| \\
& =\left|\int_{A_{x, \varepsilon}}\left(\sum_{|\gamma|<n} \frac{\partial^{\gamma} F(x)}{\gamma !} \varepsilon^{|\gamma|} y^{\gamma}+\varepsilon^{n} \sum_{|\gamma|=n} \frac{\partial^{\gamma} F(\xi)}{\gamma !} y^{\gamma}\right) \frac{1}{\varrho(y)} d \mu_{\alpha, x, \varepsilon}(y)-\varepsilon^{|\alpha|} \partial^{\alpha} F(x)\right| \\
& =\varepsilon^{n}\left|\int_{A_{x, \varepsilon}} \sum_{|\gamma|=n}\left(\frac{\partial^{\gamma} F(\xi)}{\gamma !}-\frac{\partial^{\gamma} F(x)}{\gamma !}\right) \frac{y^{\gamma}}{\varrho(y)} d \mu_{\alpha, x, \varepsilon}(y)\right|,
\end{aligned}
$$

with $\xi:=\xi(x, \varepsilon, y, f) \in[x, x+\varepsilon y]$. We split the integral into the parts over $A_{x, \varepsilon} \cap\{|y| \leq r\}$ and $A_{x, \varepsilon} \cap\{|y|>r\}$. The first integral then becomes small (for $\varepsilon \rightarrow 0$ and each fixed $r$ ) because of the uniform continuity of $\partial^{\gamma} F$ and the second becomes small (for $r \rightarrow \infty$ uniformly in $\varepsilon$ ) because of the boundedness of $\partial^{\gamma} F$, $\left|\mu_{\alpha, x, \varepsilon}\right|\left(A_{x, \varepsilon}\right) \leq 1$, and $|y|^{|\gamma|} / \varrho(y) \rightarrow 0$ for $|y| \rightarrow \infty$.

For $|\alpha|>n$ we compute similarly

$$
\begin{aligned}
\sup _{|\alpha|>n, x \in \partial K} & \frac{\left|\nu_{\alpha, x, \varepsilon}\left(f^{(0)}\right)\right|}{\varepsilon^{n}}=\sup _{|\alpha|>n, x \in \partial K}\left|\frac{1}{\varepsilon^{n}} \int_{A_{x, \varepsilon}} F(\varepsilon y+x) \frac{1}{\varrho(y)} d \mu_{\alpha, x, \varepsilon}(y)\right| \\
& =\sup _{|\alpha|>n, x \in \partial K}\left|\int_{A_{x, \varepsilon}} \sum_{|\gamma|=n}\left(\frac{\partial^{\gamma} F(\xi)}{\gamma !}-\frac{\partial^{\gamma} F(x)}{\gamma !}\right) \frac{y^{\gamma}}{\varrho(y)} d \mu_{\alpha, x, \varepsilon}(y)\right|,
\end{aligned}
$$


with $\xi:=\xi(x, \varepsilon, y, f) \in[x, x+\varepsilon y]$. By the same arguments as above we get

$$
\lim _{\varepsilon \rightarrow 0} \sup _{|\alpha|>n, x \in \partial K} \frac{\left|\nu_{\alpha, x, \varepsilon}\left(f^{(0)}\right)\right|}{\varepsilon^{n}}=0 .
$$

The proof of Proposition 5 will use duality theory (in particular, Riesz' representation theorem for duals of spaces of continuous functions to obtain the desired measures) where an improved version of LMI(1) implies a suitable continuity estimate. For this improvement we first show a very simple lemma about the Markov inequality for balls. We denote by $\mathbb{P}_{k}$ the space of polynomials of degree less or equal $k$.

Lemma 6. For each $k \in \mathbb{N}$ there is $C_{k}>0$ such that for all $Q \in \mathbb{P}_{k}$ and all $\varrho \geq 2$ we have

$$
\sum_{|\alpha| \leq k} \frac{\left|\partial^{\alpha} Q(x)\right|}{\alpha !} \varrho^{|\alpha|} \leq C_{k} \sup _{1 \leq|y-x| \leq \varrho}|Q(y)| .
$$

Proof. It is enough to show the statement for $x=0$. Since both sides of the inequality are norms on the finite dimensional space $\mathbb{P}_{k}$, there are constants $C_{k}$ such that the inequality holds for $\varrho=2$. For $\varrho>2$ we denote $Q_{\varrho}(x)=Q\left(\frac{\varrho}{2} x\right)$ and obtain

$$
\begin{aligned}
\sum_{|\alpha| \leq k}\left|\frac{\partial^{\alpha} Q(0)}{\alpha !}\right| \varrho^{|\alpha|} & =\sum_{|\alpha| \leq k}\left|\frac{\partial^{\alpha} Q_{\varrho}(0)}{\alpha !}\right| 2^{|\alpha|} \leq C_{k} \sup _{1 \leq|y| \leq 2}\left|Q_{\varrho}(y)\right| \\
& =C_{k} \sup _{\varrho / 2 \leq|y| \leq \varrho}|Q(y)| \leq C_{k} \sup _{1 \leq|y| \leq \varrho}|Q(y)| .
\end{aligned}
$$

The next lemma gives a uniform Markov inequality for the highest degree derivatives.

Lemma 7. Let $K \subseteq \mathbb{R}^{d}$ be a compact subset satisfying $\operatorname{LMI}(1)$. For all $k \in \mathbb{N}$ there is $C_{k}>0$ such that for all $\varepsilon \in(0,1), x \in \partial K, r>0$ and $P \in \mathbb{P}_{k}$ we have

$$
\sum_{|\alpha|=k}\left|\frac{\partial^{\alpha} P(0)}{\alpha !}\right| \leq \frac{C_{k}}{r^{k}} \sup \left\{|P(y)|: y \in A_{x, \varepsilon},|y| \leq r\right\} .
$$

Proof. We may assume $\varepsilon_{0}=2$ in the definition of the Markov inequality and take the sequence $c_{k}$ from there. Fix $k \in \mathbb{N}, x \in \partial K, 0<\varepsilon \leq 1, P \in \mathbb{P}_{k}$, and $0 \leq r \leq 2 / \varepsilon$. Set $Q(y):=P\left(\frac{1}{\varepsilon}(y-x)\right)$. For suitable constants $C_{k}$ (depending on $c_{k}$ and $k$ ) we get

$$
\begin{aligned}
\sum_{|\alpha|=k}\left|\frac{\partial^{\alpha} P(0)}{\alpha !}\right| r^{k} & =\sum_{|\alpha|=k}\left|\frac{\partial^{\alpha} Q(x)}{\alpha !}\right| r^{k} \varepsilon^{k} \leq C_{k} \sup _{y \in B(x, r \varepsilon) \cap K}|Q(y)| \\
& =C_{k} \sup \left\{|P(z)|: z \in \varepsilon^{-1}(K-x) \cap B(0, r)\right\} .
\end{aligned}
$$


If on the other hand $r \geq 2 / \varepsilon$, we set $\varrho=r \varepsilon$ and we get from Lemma 6 (with different constants)

$$
\begin{gathered}
\sum_{|\alpha|=k}\left|\frac{\partial^{\alpha} P(0)}{\alpha !}\right| r^{k} \leq \sum_{|\alpha| \leq k}\left|\frac{\partial^{\alpha} Q(x)}{\alpha !}\right| \varrho^{k} \leq C_{k}\{\sup |Q(y)|: 1 \leq|y-x| \leq \varrho\} \\
=C_{k} \sup \left\{|P(z)|: \varepsilon^{-1} \leq|z| \leq r\right\} \leq C_{k} \sup \left\{|P(z)|: z \in A_{\varepsilon, x},|z| \leq r\right\} .
\end{gathered}
$$

The following lemma is the main technical tool to solve the moment problem in Proposition 5.

Lemma 8. Let $K \subseteq \mathbb{R}^{d}$ be a compact subset satisfying $\mathrm{LMI}(1)$. For each family $\left(\varepsilon_{\alpha}\right)_{\alpha \in \mathbb{N}_{0}^{d}}$ of positive numbers there is a continuous and radial function $\varrho: \mathbb{R}^{d} \rightarrow$ $(0, \infty)$ with $|y|^{k}=o(\varrho(y))$ for $y \rightarrow \infty$ and each $k \in \mathbb{N}$ such that for all $x \in \partial K$, $\varepsilon \in(0,1)$, and all polynomials $P$ we have

$$
\sup _{y \in A_{x, \varepsilon}} \frac{|P(y)|}{\varrho(y)} \leq 1 \Longrightarrow \frac{\left|\partial^{\alpha} P(0)\right|}{\alpha !}<\varepsilon_{\alpha} \quad \text { for all } \alpha \in \mathbb{N}_{0}^{d} .
$$

Proof. Let $\left(\varepsilon_{\alpha}\right)_{\alpha \in \mathbb{N}_{0}^{d}}$ be given. For a suitable increasing sequence $R_{k} \rightarrow \infty$ we define a radial weight function $\varrho: \mathbb{R}^{d} \rightarrow(0, \infty)$ by $\varrho(0)=\varepsilon_{0} / 3$ and

$$
\varrho(x)=|x|^{k-1} \quad \text { for } R_{k-1}<|x| \leq R_{k} .
$$

We will construct the sequence $R_{k}$ so that $\varrho$ satisfies slightly more than the assertion of the lemma. Afterwards it is easy to find a continuous modification.

Proceeding recursively, we will show that $\left(R_{k}\right)_{k}$ can be found such that for all polynomials $P \in \mathbb{P}_{k}, x \in \partial K$, and $\varepsilon \in(0,1)$ we have

$$
\sup _{y \in A_{x, \varepsilon},|y| \leq R_{k}} \frac{|P(y)|}{\varrho(y)} \leq 1+\frac{1}{k+1} \Longrightarrow \frac{\left|\partial^{\alpha} P(0)\right|}{\alpha !}<\varepsilon_{\alpha}
$$

for all $|\alpha| \leq k$. Since $\varrho(0)<\varepsilon_{0} / 2$, this is true for $k=0$. We assume that $R_{0}, \ldots, R_{k-1}$ are constructed so that

$$
\sup _{y \in A_{x, \varepsilon},|y| \leq R_{k-1}} \frac{|P(y)|}{\varrho(y)} \leq 1+\frac{1}{k} \Longrightarrow \frac{\left|\partial^{\alpha} P(0)\right|}{\alpha !}<\varepsilon_{\alpha}
$$

for all $|\alpha| \leq k-1$ and $P \in \mathbb{P}_{k-1}$. From Lemma 7 we get $C_{k}>0$ such that, for all $P \in \mathbb{P}_{k}, x \in \partial K, \varepsilon \in(0,1)$, and $R>0$,

$$
\sum_{|\alpha|=k} \frac{\left|\partial^{\alpha} P(0)\right|}{\alpha !} \leq \frac{C_{k}}{R} \sup _{y \in A_{x, \varepsilon},|y| \leq R} \frac{|P(y)|}{R^{k-1}} .
$$

We choose $R_{k}>R_{k-1}$ such that

$$
\frac{C_{k}}{R_{k}}<\min \left\{\varepsilon_{\alpha} / 2:|\alpha|=k\right\} \quad \text { and } \quad \frac{C_{k}}{R_{k}}<\frac{\varepsilon_{0}}{3(k+1)^{2} R_{k-1}^{k}} .
$$


If now $P \in \mathbb{P}_{k}$ satisfies

$$
\sup _{y \in A_{x, \varepsilon},|y| \leq R_{k}} \frac{|P(y)|}{\varrho(y)} \leq 1+\frac{1}{k+1},
$$

we apply (3.1) for $R=R_{k}$. Since $\varrho(y)$ is increasing we get, for $|\alpha|=k$,

$$
\frac{\left|\partial^{\alpha} P(0)\right|}{\alpha !} \leq \frac{C_{k}}{R_{k}} \sup _{y \in A_{x, \varepsilon},|y| \leq R_{k}} \frac{|P(y)|}{R_{k}^{k-1}}<\frac{\varepsilon_{\alpha}}{2} \sup _{y \in A_{x, \varepsilon},|y| \leq R_{k}} \frac{|P(y)|}{\varrho(y)} \leq \varepsilon_{\alpha} .
$$

Let $P_{k}$ be the homogeneous part of $P$ of degree $k$. Using $\varrho(y) \leq R_{k}^{k-1}$ for $|y| \leq R_{k}$ and again (3.1) for $R=R_{k}$ we obtain

$$
\begin{aligned}
& \sup _{y \in A_{x, \varepsilon},|y| \leq R_{k-1}} \frac{\left|\left(P-P_{k}\right)(y)\right|}{\varrho(y)} \leq \sup _{y \in A_{x, \varepsilon},|y| \leq R_{k}} \frac{|P(y)|}{\varrho(y)}+\sup _{y \in A_{x, \varepsilon},|y| \leq R_{k-1}} \frac{\left|P_{k}(y)\right|}{\varrho(0)} \\
& \leq 1+\frac{1}{k+1}+\frac{3}{\varepsilon_{0}} \sum_{|\alpha|=k} \frac{\left|\partial^{\alpha} P(0)\right|}{\alpha !} R_{k-1}^{k} \leq 1+\frac{1}{k+1}+\frac{3 R_{k-1}^{k}}{\varepsilon_{0}} \frac{C_{k}}{R_{k}} \sup _{y \in A_{x, \varepsilon},|y| \leq R_{k}} \frac{|P(y)|}{R_{k}^{k-1}} \\
& \leq 1+\frac{1}{k+1}+\frac{1}{(k+1)^{2}} \sup _{y \in A_{x, \varepsilon},|y| \leq R_{k}} \frac{|P(y)|}{\varrho(y)} \leq 1+\frac{1}{k} .
\end{aligned}
$$

Using the induction assumption we conclude

$$
\frac{\left|\partial^{\alpha} P(0)\right|}{\alpha !}=\frac{\left|\partial^{\alpha}\left(P-P_{k}\right)(0)\right|}{\alpha !}<\varepsilon_{\alpha} \quad \text { for }|\alpha|<k .
$$

Proof of Proposition 5. Consider the space

$$
\varphi\left(\mathbb{N}_{0}^{d}\right)=\left\{x: \mathbb{N}_{0}^{d} \rightarrow \mathbb{C}:\left\{\alpha \in \mathbb{N}_{0}^{d}: x_{\alpha} \neq 0\right\} \text { finite }\right\}
$$

as the locally convex direct sum of $\mathbb{N}_{0}^{d}$ copies of $\mathbb{C}$. It has the finest locally convex topology (that is, every seminorm on it is continuous or, equivalently, every absorbing absolutely convex set is a neighbourhood of 0) and it is the dual of the product $\omega\left(\mathbb{N}_{0}^{d}\right)=\mathbb{C}^{\mathbb{N}_{0}^{d}}$ with the product topology where $x \in \varphi\left(\mathbb{N}_{0}^{d}\right)$ acts on $\omega\left(\mathbb{N}_{0}^{d}\right)$ by $y \mapsto\langle x, y\rangle=\sum_{\beta} x_{\beta} y_{\beta}$. The set

$$
L=\left\{\left(\alpha ! \delta_{\alpha, \beta}\right)_{\beta \in \mathbb{N}_{0}^{d}}: \quad \alpha \in \mathbb{N}_{0}^{d}\right\} \subseteq \omega\left(\mathbb{N}_{0}^{d}\right)
$$

is compact in $\omega\left(\mathbb{N}_{0}^{d}\right)$ by Tychonov's theorem and thus, the polar $L^{\circ}=\left\{x \in \varphi\left(\mathbb{N}_{0}^{d}\right)\right.$ : $|\langle x, y\rangle| \leq 1$ for all $y \in L\}$ is a 0-neighbourhood in $\varphi\left(\mathbb{N}_{0}^{d}\right)$ because $L^{\circ}$ is absolutely convex and absorbing. Therefore, there is a family $\left(\varepsilon_{\alpha}\right)_{\alpha \in \mathbb{N}_{0}^{d}}$ of positive numbers such that $U=\left\{\left(\lambda_{\alpha}\right)_{\alpha \in \mathbb{N}_{0}^{d}} \in \varphi\left(\mathbb{N}_{0}^{d}\right):\left|\lambda_{\alpha}\right|<\varepsilon_{\alpha}\right\}$ is a 0-neighbourhood in $\varphi\left(\mathbb{N}_{0}^{d}\right)$ with $U \subseteq L^{\circ}$. For the weight function $\varrho$ from Lemma $8, x \in K$ and $\varepsilon \in(0,1)$ we consider the spaces

$$
C_{x, \varepsilon}:=\left\{f \in \mathscr{C}\left(A_{x, \varepsilon}\right): \lim _{y \rightarrow \infty} \frac{|f(y)|}{\varrho(y)}=0\right\}
$$


equipped with the weighted sup-norm $\|f\|_{x, \varepsilon}=\sup \left\{|f(y)| / \varrho(y): y \in A_{x, \varepsilon}\right\}$, and denote the corresponding unit ball by $B_{x, \varepsilon}$. In a functional analytic form, Lemma 8 says that the continuous linear mapping

$$
T_{x, \varepsilon}: \varphi\left(\mathbb{N}_{0}^{n}\right) \rightarrow C_{x, \varepsilon},\left(\lambda_{\alpha}\right)_{\alpha \in \mathbb{N}_{0}^{d}} \mapsto\left(y \mapsto \sum_{\alpha \in \mathbb{N}_{0}^{d}} \lambda_{\alpha} y^{\alpha}\right)
$$

satisfies $T_{x, \varepsilon}^{-1}\left(B_{x, \varepsilon}\right) \subseteq U$ for each $x \in K$ and each $\varepsilon \in(0,1)$. Since the map $C_{x, \varepsilon} \rightarrow \mathscr{C}_{0}\left(A_{x, \varepsilon}\right), f \mapsto f / \varrho$ is an isometry Riesz' representation theorem yields

$$
C_{x, \varepsilon}^{\prime}=\left\{\frac{1}{\varrho} \mu: \mu \text { a regular finite Borel measure on } A_{x, \varepsilon}\right\},
$$

and the unit ball of $C_{x, \varepsilon}^{\prime}$ is

$$
B_{x, \varepsilon}^{\circ}=D_{x, \varepsilon}:=\left\{\frac{1}{\varrho} \mu:|\mu|\left(A_{x, \varepsilon}\right) \leq 1\right\} .
$$

By Alaoğlu's theorem, this dual unit ball is weak*-compact, and $T_{x, \varepsilon}^{t}: C_{x, \varepsilon}^{\prime} \rightarrow$ $\omega\left(\mathbb{N}_{0}^{d}\right)$ is weak ${ }^{*}$-weak ${ }^{*}$ continuous (this is true for the transposed of every operator) so that the image $T_{x, \varepsilon}^{t}\left(D_{x, \varepsilon}\right)$ is weak*-closed. The bipolar theorem yields $T_{x, \varepsilon}^{t}\left(D_{x, \varepsilon}\right)=T_{x, \varepsilon}^{t}\left(D_{x, \varepsilon}\right)^{\circ \circ}$. Applying the general fact that $S(A)^{\circ}=\left(S^{t}\right)^{-1}\left(A^{\circ}\right)$ to $S=T^{t}$ and $A=D_{x, \varepsilon}=B_{x, \varepsilon}^{\circ}$ we obtain from $T^{t t}=T$ and $B_{x, \varepsilon}^{\circ \circ}=B_{x, \varepsilon}$ that

$$
T_{x, \varepsilon}^{t}\left(D_{x, \varepsilon}\right)=T_{x, \varepsilon}^{t}\left(D_{x, \varepsilon}\right)^{\circ \circ}=T_{x, \varepsilon}^{-1}\left(B_{x, \varepsilon}\right)^{\circ} \supseteq U^{\circ} \supseteq L^{\circ \circ} \supseteq L .
$$

Since $z=\left(\alpha ! \delta_{\alpha, \beta}\right)_{\beta \in \mathbb{N}_{0}^{d}} \in L$ we thus find a measure $\mu=\mu_{\alpha, x, \varepsilon}$ on $A_{x, \varepsilon}$ with total variation bounded by 1 such that $z=T_{x, \varepsilon}^{t}(\mu / \varrho)$. This implies the assertion of Proposition 5 since, for the canonical unit vectors $e_{\gamma}=\left(\delta_{\gamma, \beta}\right)_{\beta \in \mathbb{N}_{0}^{d}}$,

$$
\left\langle T_{x, \varepsilon}^{t}(\mu / \varrho), e_{\gamma}\right\rangle=\mu / \varrho\left(T_{x, \varepsilon} e_{\gamma}\right)=\int y^{\gamma} / \varrho(y) d \mu(y)
$$

\section{References}

[1] Bierstone, E. And Milman, P. D.: Geometric and differential properties of subanalytic sets. Ann. of Math. (2) 147 (1998), no. 3, 731-785.

[2] Borel, E.: Sur quelques points de la théorie des fonctions. Ann. Sci. École Norm. Sup. (3) 12 (1895), 9-55.

[3] Bos, L. P. And Milman, P. D.: Sobolev-Gagliardo-Nirenberg and Markov type inequalities on subanalytic domains. Geom. Funct. Anal. 5 (1995), no. 6, 853-923.

[4] Bourbaki, N.: Espaces vectoriels topologiques. Chapitres 1-5. Masson, Paris, 1981.

[5] Fefferman, C. And Ricci, F.: Some examples of $C^{\infty}$ extension by linear operators. Rev. Mat. Iberoam. 28 (2012), no. 1, 297-304.

[6] FrERICK, L.: Extension operators for spaces of infinite differentiable Whitney jets. J. Reine Angew. Math. 602 (2007), 123-154. 
[7] Frerick, L., Jordá, E. And Wengenroth, J.: Tame linear extension operators for smooth Whitney functions. J. Funct. Anal. 261 (2011), no. 3, 591-603.

[8] Jonsson, A., Suögren, P. And Wallin, H.: Hardy and Lipschitz spaces on subsets of $\mathbb{R}^{n}$. Studia Math. 80 (1984), no. 2, 141-166.

[9] Malgrange, B.: Ideals of differentiable functions. Tata Institute of Fundamental Research Studies in Mathematics 3, Tata Institute of Fundamental Research, Bombay; Oxford University Press, London, 1967.

[10] Meise, R. And Vogt, D.: Introduction to functional analysis. Oxford Graduate Texts in Mathematics 2, The Clarendon Press, Oxford University Press, New York, 1997.

[11] Mitjagin, B. S.: Approximate dimension and bases in nuclear spaces. Uspehi Mat. Nauk 16 (1961), no. 4 (100), 63-132.

[12] Pawłucki, W. and Pleśniak, W.: Markov's inequality and $C^{\infty}$ functions on sets with polynomial cusps. Math. Ann. 275 (1986), no. 3, 467-480.

[13] PawŁucki, W. And Pleśniak, W.: Extension of $C^{\infty}$ functions from sets with polynomial cusps. Studia Math. 88 (1988), no. 3, 279-287.

[14] Pleśniak, W.: Markov's inequality and the existence of an extension operator for $C^{\infty}$ functions. J. Approx. Theory 61 (1990), no. 1, 106-117.

[15] Rogers, L. G.: Degree-independent Sobolev extension on locally uniform domains. J. Funct. Anal. 235 (2006), no. 2, 619-665.

[16] Seeley, R. T.: Extension of $C^{\infty}$ functions defined in a half space. Proc. Amer. Math. Soc. 15 (1964), 625-626.

[17] Stein, E. M.: Singular integrals and differentiability properties of functions. Princeton Mathematical Series 30, Princeton University Press, Princeton, NJ, 1970.

[18] Tidten, M.: Fortsetzungen von $C^{\infty}$-Funktionen, welche auf einer abgeschlossenen Menge in $\mathbb{R}^{n}$ definiert sind. Manuscripta Math. 27 (1979), no. 3, 291-312.

[19] Whitney, H.: Analytic extensions of differentiable functions defined in closed sets. Trans. Amer. Math. Soc. 36 (1934), no. 1, 63-89.

Received April 22, 2014.

LeOnhard FreRICK: Fachbereich IV - Mathematik, Universität Trier, 54286 Trier, Germany.

E-mail: frerick@uni-trier.de

Enrique Jordá: Departamento de Matemática Aplicada, Escuela Politécnica Superior de Alcoy, Universidad Politécnica de Valencia, Plaza Ferrándiz y Carbonell 2, 03801 Alcoy (Alicante), Spain.

E-mail: ejorda@mat.upv.es

Jochen Wengenroth: Fachbereich IV - Mathematik, Universität Trier, 54286 Trier, Germany.

E-mail: wengenroth@uni-trier.de

The research of the first and second named authors was partially supported MINECO, Project MTM2013-43540-P. 